\title{
Flow Properties of Polymer Solutions. I. Temperature Dependence of Stresses Following Sudden Start and Cessation of Steady Shear Flow*
}

\author{
Kunihiro Osaki, Yoshiyuki EINAGA, ${ }^{* *}$ Nobuhiro Yamada, ${ }^{* * *}$ \\ and Michio Kurata \\ Institute for Chemical Research, Kyoto University, \\ Uji, Kyoto 611, Japan.
}

(Received August 20, 1973)

\begin{abstract}
Following sudden start and cessation, respectively, of steady shear flow, stresses, $\kappa \bar{\eta}(t, \kappa)$ and $\kappa \tilde{\eta}(t, \kappa)$, were measured for a $20-\%$ solution of polystyrene in chlorinated biphenyl. Here $\kappa$ is the rate of shear and $t$ is the time after sudden change of rate of shear. Measurements were performed at every $5^{\circ} \mathrm{C}$ starting from $30^{\circ} \mathrm{C}$ up to $50^{\circ} \mathrm{C}$ with a rheometer of the cone-and-plate type in the range of rate of shear $1 \times 10^{-4} \sim 5 \times 10^{-2} \mathrm{sec}^{-1}$. Measured results included behaviors characteristic of high rates of shear such as stress overshoot as well as those characteristic of low rates of shear. The accuracy of data as examined with linear viscoelasticity relations at low rates of shear was satisfactory. A method of reduced variables with respect to temperature $T$, time $t$, and rate of shear $\kappa$ was revealed to apply to stress development function $\bar{\eta}(t, \kappa)$ and stress decay function $\tilde{\eta}(t, \kappa): \bar{\eta}(t, \kappa) / \eta^{0}$ and $\tilde{\eta}(t, \kappa) / \eta^{0}$ obtained at various temperatures were unique functions of two variables $t / a_{\mathrm{T}}$ and $\kappa a_{\mathrm{T}}$, where $\eta^{0}$ is the zero-shear viscosity and $a_{\mathrm{T}}$ is the shift factor usually employed in the time-temperature reduction for functions in linear viscoelasticity.

KEY WORDS Steady Shear / Stress Overshoot / Stress Decay /

Reduced Variables / Shear Rate / Polystyrene /
\end{abstract}

In this series of papers, we will study flow properties of polymer solutions in the transient states following sudden start and cessation of steady shear flow. The stress development at the beginning of steady shear flow and the stress decay after sudden stop of the steady shear flow $^{1-5}$ have often been investigated in regard to the construction of phenomenological models for nonlinear viscoelasticity. Some of the proposed models have attained fair success in describing the experimental results. ${ }^{6}$ On the other hand, relaxation moduli obtained under large strain have been shown to depend on the magnitude of strain. ${ }^{7-10}$ This result has suggested that a group of simple constitutive equations

* Request for Reprints should be addressed to M. Kurata.

** Present address: Department of Polymer Science, Osaka University, Toyonaka.

*** Department of Industrial Chemistry, Kyoto University. Present address: Nippon Tokushu Kento, Ltd., Kisshoin, Minami, Kyoto. based on strain-rate-dependent relaxation spectrum $^{6,11}$ may not be applied to the stress under strains of a discontinuous nature as functions of time although they may be applied to the stress development and the stress decay at the start and the cessation, respectively, of steady shear flow. Investigation of the stress decay after application of double-step $\operatorname{strain}^{8,12}$ has. revealed that constitutive equations based on strain-dependent relaxation spectra ${ }^{6,8,13}$ may be applied to strains which are discontinuous as functions of time unless the strains are very large. Thus it has become interesting to compare the stresses at the start and the cessation of steady shear flow with strain-dependent relaxation moduli.

In the present paper, we examine the effect of varying temperature on the stress development and the stress decay. Let us define two viscoelastic functions $\bar{\eta}(t, \kappa)$ and $\tilde{\eta}(t, \kappa)$ by dividing the stresses at time $t$ after sudden start and 
Flow Properties of Polymer Solutions. I.

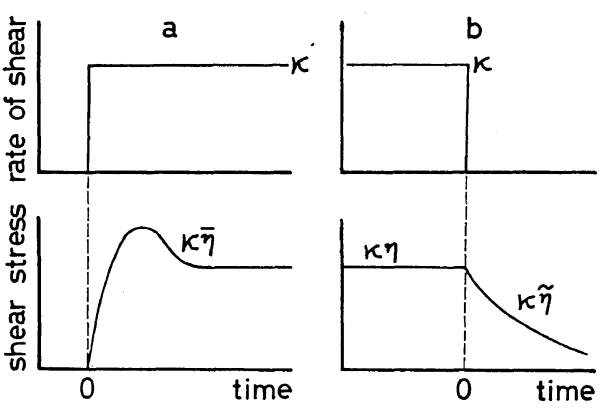

Figure 1. Illustration of stress development (a) and stress decay (b) on start and cessation of steady shear flow. Upper panels represent rate of shear and lower panels stress as functions of time.

cessation, respectively, of steady shear flow by the rate of shear $\kappa$ (see Figure 1). It may be noted that $\bar{\eta}(\infty, \kappa)=\tilde{\eta}(0, \kappa) \equiv \eta(\kappa)$ is the steadyshear viscosity and $\eta^{0} \equiv \eta(0)$ is the zero-shear viscosity. At the limit of zero-rate of shear, functions $\bar{\eta}(t) \equiv \bar{\eta}(t, 0)$ and $\tilde{\eta}(t) \equiv \tilde{\eta}(t, 0)$ may be related to other viscoelastic functions through linear viscoelasticity relations, e.g.,

$$
\bar{\eta}(t)=\eta^{0}-\tilde{\eta}(t)=\int_{0}^{t} G\left(t^{\prime}\right) \mathrm{d} t^{\prime}
$$

where $G(t)$ is the relaxation modulus in linear viscoelasticity. ${ }^{14}$

A time-temperature reduction rule has proved itself very effective in describing the temperature dependence of viscoelastic functions in linear viscoelasticity of polymeric systems whose internal structures do not vary much with varying temperature. ${ }^{15}$ According to the time-temperature reduction rule, a single composite curve is obtained if $\bar{\eta}(t) / \eta^{0}$ or $\tilde{\eta}(t) / \eta^{0}$ obtained at various temperatures are plotted against $t / a_{\mathrm{T}}$ where the shift factor $a_{\mathrm{T}}=\eta^{0} T_{0} \rho_{0} / \eta_{0}^{0} T \rho$ is evaluated from the viscosity $\eta^{0}$ and the density $\rho$ at the temperature $T^{\circ} \mathrm{K}$ and corresponding quantities $\eta_{0}^{0}$ and $\rho_{0}$ at the reference temperature $T_{0}$ chosen arbitrarily. Since the factor $T_{0} \rho_{0} / T \rho$ is rather insensitive to the variation of temperature, the temperature dependence of the viscosity approximately represents the temperature dependence of the shift factor. Due to the phenomenological relations of linear viscoelasticity, the time-temperature reduction rule may be applied to any viscoelastic functions if it applies to any one viscoelastic function in the temperature range in question.

In the case of nonlinear viscoelasticity, analogous methods of reduced variables have been found to be applicable to a few simple quantities such as strain-dependent relaxation modulus and stresses in steady shear flow. The straindependent tensile relaxation modulus for crosslinked polymers measured at a constant strain obeys the time-temperature reduction rule with the same shift factor $a_{\mathrm{T}}$ as evaluated in linear viscoelasticity. ${ }^{16}$ The same has been found to be true for the strain-dependent shear relaxation modulus for a concentrated polymer solution. ${ }^{10}$ On the other hand, a shear rate-temperature reduction rule has been proved to apply to stresses in steady shear flow for some polymeric systems. ${ }^{15}$ As exemplified with the shear stress $\sigma$, a reduced steady-shear viscosity $\eta(\kappa) / \eta^{0}\left(=\sigma / \kappa \eta^{0}\right)$ obtained at various temperatures is a unique function of a reduced rate of shear $\kappa a_{T}$, where $a_{\mathrm{T}}$ is independent of $\kappa$ and is the same as in linear viscoelasticity in most cases. It is to be noted that the successful applicability of the method of reduced variables for one viscoelastic quantity does not necessarily mean its applicability to other quantities in the case of nonlinear viscoelasticity.

The quantities $\bar{\eta}(t, \kappa)$ and $\tilde{\eta}(t, \kappa)$ are functions of two variables $t$ and $\kappa$, to which the method of reduced variables with respect to temperature is applicable in the limiting cases of linear viscoelasticity and steady flow, respectively. The question is whether a simple reduction rule is applicable to these functions of two variables. If a simple reduction rule is discovered, it may be employed to obtain data over wide ranges of variables $t$ and $\kappa$ by changing the temperature in further studies on analogous polymer solutions. The form (or lack) of a reduction rule will put a restriction on possible constitutive equations as discussed in the following paper.

In the first paper of this series, we describe measurements of $\bar{\eta}(t, \kappa)$ and $\tilde{\eta}(t, \kappa)$ and examine time-temperature and shear rate-temperature reduction rules for a concentrated polymer solution. 


\section{EXPERIMENTAL}

Measurements of the stress development and the stress decay on start and sudden stop, respectively, of steady shear flow were performed with a rheometer of the cone-and-plate type. Details of the apparatus will be reported elsewhere. ${ }^{17}$ In the present paper are given outlines of the apparatus and of the preliminary tests relevant to the present study.

The sample holder of this apparatus consists of a disk plate and a cone with its apex on the surface of the plate at its center. The sample liquid is sandwiched in the gap between these parts. The cone is suspended with a steel torsion wire, whose torsional displacement, measured with a differential transformer, gives the torque exerted to the cone from the liquid. The plate can be rotated at various velocities with a driving system through an electromagnetic clutch system. The clutch system originates sudden start and cessation of steady rotation by connecting and disconnecting, respectively, the plate and the driving system. On disconnection of the clutch, a brake connected electrically to the clutch prevents the inertial motion of the plate. The shear stress and the rate of shear of the sample are calculated from the torque and the rotation velocity, respectively. The plate may be driven in a sinusoidally oscillatory rotation of variable amplitude or in a superimposed oscillation on steady rotation for measurements of complex modulus or complex modulus with superimposed steady shear, respectively.

The proper functioning of the clutch system was examined by recording a spot of light reflected from a mirror attached to the plate on a moving photographic film. Discontinuous or vibrational motion of the plate was not observed on connection or disconnection of the clutch; constant rotation velocity and sudden stop of the rotation, respectively, were attained within $0.01 \mathrm{sec}$ at any rotation velocity. Since no servo-mechanism was employed in the stressdetecting device, the displacement of the cone was not negligible with regard to the proper deformation of the sample if the torsion wire was too thin. Appropriate wires were chosen for each sample by performing experiments with several different wires. The experiments at various rates of shear were performed only with those wires for which the results were in agreement within scattering of the data with the extrapolated results at the limit of infinitely large wire constant (or no displacement of the wire). The precision of the results, as examined with the viscosities of a few Newtonian liquids, was satisfactory.

Measurements of the stress development and the stress decay, or $\bar{\eta}(t, \kappa)$ and $\tilde{\eta}(t, \kappa)$, respective$1 y$, were performed for a $20-\%$ solution of polystyrene in Aroclor 1248. The polystyrene was the standard sample 14a of molecular weight $1.80 \times 10^{6}$ supplied from Pressure Chemical Company and Aroclor 1248 was partially chlorinated biphenyl supplied from Monsanto Chemical Company. The solution has been studied with reference to its nonlinear behavior ${ }^{9,10,12,18}$ as well as its linear behavior. ${ }^{19,20}$ The experiments were carried out at steps of $5^{\circ} \mathrm{C}$ in the range from 30 to $50^{\circ} \mathrm{C}$. The range of rate of shear $\kappa$ was approximately from $10^{-4}$ to $5 \times 10^{-2} \mathrm{sec}^{-1}$. The highest rate of shear was determined by the fact that the sample came out of the sample holder at higher rate of shear.

\section{RESULTS AND DISCUSSION}

\section{Stress Development and Stress Decay Functions}

The stress development function $\bar{\eta}(t, \kappa)$ and the stress decay function $\tilde{\eta}(t, \kappa)$ obtained for the polystyrene solution at $30^{\circ} \mathrm{C}$ are plotted against time $t$ in Figures 2 and 3, respectively. The results for the rate of shear lower than $1.70 \times$ $10^{-4} \mathrm{sec}^{-1}$ are not shown because $\bar{\eta}(t, \kappa)$ as well as $\widetilde{\eta}(t, \kappa)$ is independent of $\kappa$ in this case and is in agreement with that for $\kappa=1.70 \times 10^{-4}$.

At low rates of shear, $\bar{\eta}(t, \kappa)$ increases with increasing time and decreases with rate of shear. At high rates of shear, the phenomenon of stress overshoot is observed in Figure 2, i.e., $\bar{\eta}(t, \kappa)$ increases rapidly first as $t$ increases, passes a maximum and then decreases to approach the value $\eta(\kappa)$ for the steady state. The maximum is located at a longer time scale and the maximum value is lower for lower rates of shear. It may be noted that $\bar{\eta}(t, \kappa)$ for any rate of shear is equal within experimental scattering to that for the lowest rate of shear up to the time corresponding to approximately $\kappa t=0.7$ and then 
Flow Properties of Polymer Solutions. I.

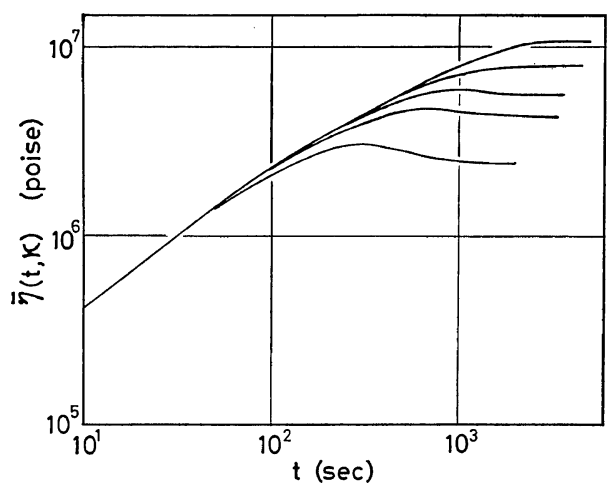

Figure 2. Stress development function $\bar{\eta}(t, \kappa)$ plotted against time $t$ for solution of polystyrene in chlorinated biphenyl at $30^{\circ} \mathrm{C}$. Rates of shear $\kappa$ are $1.70 \times 10^{-4}, 8.65 \times 10^{-4}, 2.30 \times 10^{-3}, 3.61 \times 10^{-3}$, and $8.86 \times 10^{-3} \mathrm{sec}^{-1}$ from top to bottom.

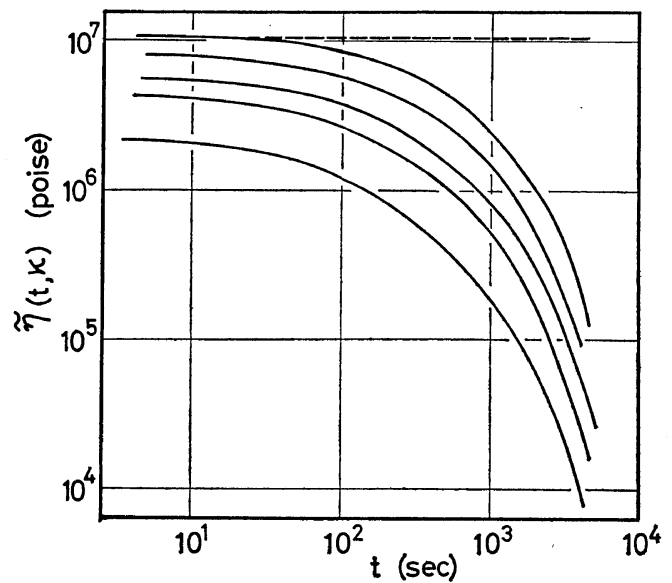

Figure 3. Stress decay function $\tilde{\eta}(t, \kappa)$ plotted against time $t$ for solution of polystyrene in chlorinated biphenyl at $30^{\circ} \mathrm{C}$. Rates of shear $\kappa$ are $1.70 \times 10^{-4}, 8.65 \times 10^{-4}, 2.30 \times 10^{-3}, 3.61 \times 10^{-3}$, and $8.86 \times 10^{-3} \mathrm{sec}^{-1}$ from top to bottom. Dashed line represents $\bar{\eta}(t, \kappa)+\tilde{\eta}(t, \kappa)$ for $\kappa=1.70 \times 10^{-4}$, where $\bar{\eta}(t, k)$ is stress development function of Figure 2.

becomes lower than that for the lowest $\kappa$. In other words, the nonlinear behavior is not observed for any value of $\kappa$ up to the time $0.7 / \kappa$ for this polymer solution.

The stress decay function $\tilde{\eta}(t, \kappa)$ as shown in Figure 3 is a continuously decreasing function of time at any rate of shear. The curves of $\log \tilde{\eta}(t, \kappa)$ plotted against $\log t$ for various values of $\kappa$ are very similar to one another but a close inspection reveals that the relative rate of decrease of $\tilde{\eta}(t, \kappa)$ (i.e., $-\partial \log \tilde{\eta} / \partial \log t)$ is the larger, the higher the rate of shear over a wide range of time scale but over very long times. The dashed line represents the sum $\bar{\eta}(t, \kappa)+$ $\tilde{\eta}(t, \kappa)$ calculated for the lowest rate of shear, $1.70 \times 10^{-4} \mathrm{sec}^{-1}$. The linear viscoelasticity relation of eq 1 indicates that the sum is equal to the zero-shear viscosity $\eta^{0}$. It is seen that the sum is not only independent of $t$ but equal to the value of $\tilde{\eta}(t, \kappa)$ for the lowest rate of shear at the limit of $t \rightarrow 0$. This result implies that $\bar{\eta}(t, \kappa)$ and $\tilde{\eta}(t, \kappa)$ at $\kappa=1.70 \times 10^{-4} \sec ^{-1}$ are consistent with each other in view of the linear viscoelasticity.

Figure 4 shows the comparison of the reported result ${ }^{10}$ for relaxation modulus $G(t)$ and $-\partial \tilde{\eta}(t, \kappa) / \partial t$ calculated for the lowest rate of shear in Figure 3. The phenomenological relation eq 1 requires these quantities to be equal to each other if these quantities are consistent in regard to the linear viscoelasticity. It is obvious that $G(t)$ (thick line) and $-\partial \tilde{\eta}(t, \kappa) / \partial t$ (circles) are in good agreement over the whole range of time scale studied. This result and that of Figure 3 implies that the results of $\bar{\eta}(t, \kappa)$ and $\tilde{\eta}(t, \kappa)$ at low rates of shear are consistent with the result of $G(t)$ measured with another apparatus. Since the measurements of $\bar{\eta}(t, \kappa)$ and $\tilde{\eta}(t, \kappa)$ are easier at higher rates of

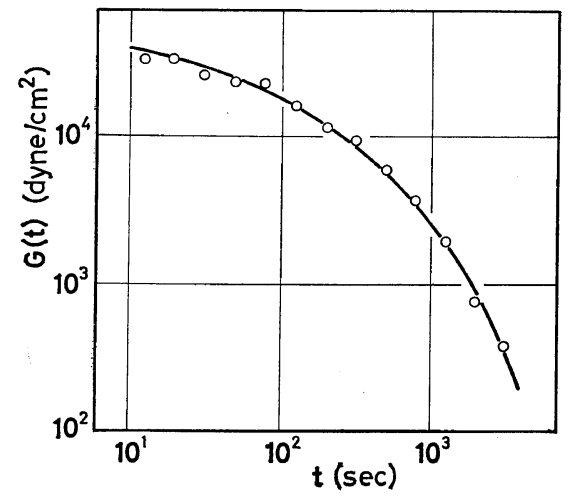

Figure 4. Relaxation modulus $G(t)$ plotted against time $t$ for solution of polystyrene in chlorinated biphenyl. The thick line represents the reported result ${ }^{10}$ and the circles the result obtained from the stress decay function $\tilde{\eta}(t, \kappa)$ of Figure 3 for $\kappa=1.70 \times 10^{-4} \mathrm{sec}^{-1}$ with $G(t)=-\partial \tilde{\eta}(t, \kappa) / \partial t$. 
shear, the precision of the data of Figures 2 and 3 may be fairly good at any rate of shear.

Analogous results were obtained for $\bar{\eta}(t, \kappa)$ and $\tilde{\eta}(t, \kappa)$ at higher temperatures. The effect of raising the temperature may be summarized as follows. Both $\bar{\eta}(t, \kappa)$ and $\tilde{\eta}(t, \kappa)$ decrease as temperature increases. $\bar{\eta}(t, \kappa)$ approaches the value at steady state more rapidly and $\tilde{\eta}(t, \kappa)$ decays more rapidly at higher temperature. The linear viscoelastic behavior persists up to higher rates of shear and the stress overshoot is observed only at higher rates of shear than those of Figure 2. However, the property that $\bar{\eta}(t, \kappa)$ for $t<0.7 / \kappa$ is independent of $\kappa$ is true at any temperature investigated.

Temperature Dependences of $\bar{\eta}(t, \kappa)$ and $\tilde{\eta}(t, \kappa)$

Figure 5 shows the steady-shear viscosity $\eta(\kappa)$ obtained at various temperatures. The viscosity is independent of the rate of shear $\kappa$ at low rates of shear and then decreases with increasing $\kappa$ at higher rates of shear at each temperature. The decrease of $\eta(\kappa)$ begins at higher rates of shear when the temperature is higher. The zero-shear viscosity decreases more than a decade with an increment of temperature of $20^{\circ} \mathrm{C}$.

The result of reduced variables with respect to temperature and rate of shear is shown in Figure 6 (group of points at the top) for the steady-shear viscosity of Figure 5. In Figure 6, $\eta(\kappa) / \eta^{0}$ obtained at various temperatures are plotted against $\kappa a_{\mathrm{T}}$, where $a_{\mathrm{T}}$ is taken as $\eta^{0} / \eta_{0}{ }^{0}$ with neglect of the slight variation of $T_{0} \rho_{0} / T \rho$ and the reference temperature indicated by subscript zero is chosen as $30^{\circ} \mathrm{C}$. It may be obvious that the shear rate-temperature reduction rule is applicable to this system. In other words, $\bar{\eta}(\infty, \kappa) / \eta^{0}=\tilde{\eta}(0, \kappa) / \eta^{0}\left(=\eta(\kappa) / \eta^{0}\right)$ obtained at various temperatures is a unique function of the reduced rate of shear $\kappa a_{\mathrm{T}}$. It has also been observed that quantities $\bar{\eta}(t, 0) / \eta^{0}$ and $\tilde{\eta}(t, 0) / \eta^{0}$ obtained at various temperatures are unique functions of a reduced time $t / a_{\mathrm{T}}$ in agreement with the results in linear viscoelasticity reported previously. ${ }^{19}$

In examining temperature dependences of $\bar{\eta}(t, \kappa)$ or $\tilde{\eta}(t, \kappa)$ at finite values of $t$ and $\kappa$, one can not find a simple relation by comparing $\bar{\eta}\left(t, \kappa=\kappa_{0}\right)$ or $\tilde{\eta}\left(t, \kappa=\kappa_{0}\right)$ obtained at various tem-

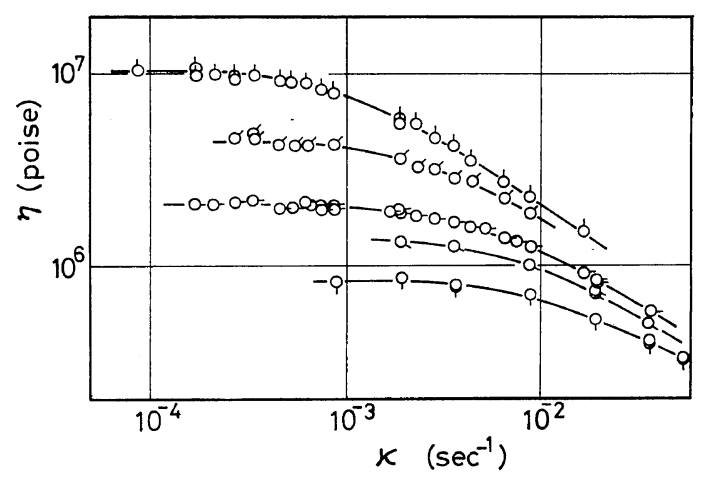

Figure 5. Steady-shear viscosity $\eta(\kappa)$ plotted against rate of shear $\kappa$ for a solution of polystyrene in chlorinated biphenyl. Various directions of "pips" represent temperatures: "Pip up" signifies $30^{\circ} \mathrm{C}$ and successive $45^{\circ}$ rotations clockwise correspond to $35,40,45$, and $50^{\circ} \mathrm{C}$, respectively.

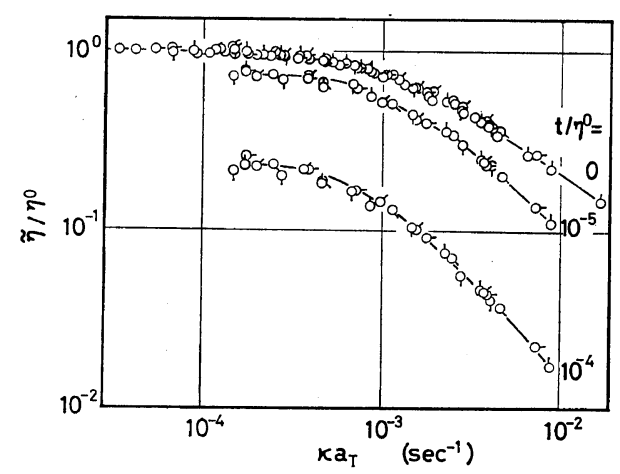

Figure 6. Reduced stress decay function $\tilde{\eta}(t, \kappa) / \eta^{0}$ plotted against reduced rate of shear $\kappa a_{T}$ for solution of polystyrene in chlorinated biphenyl. Reference temperature is $30^{\circ} \mathrm{C}$ and various directions of "pips" represent temperatures as indicated in Figure 5. Reduced times $t / \eta^{0}$ are $0,10^{-5}$, and $10^{-4} \mathrm{sec} /$ poise, respectively, from top to bottom.

peratures where $\kappa_{0}$ represents any constant rate of shear independent of temperature; $\bar{\eta}(t, 8.86 \times$ $10^{-3}$ ), for example, does exhibit a maximum at $30^{\circ} \mathrm{C}$ while it increases continuously with increasing $t$ at $50^{\circ} \mathrm{C}$. It is also impossible to find a simple reduction rule for $\bar{\eta}\left(t=t_{0}, \kappa\right)$ or $\tilde{\eta}\left(t=t_{0}, \kappa\right)$ with respect to temperature and rate of shear where $t_{0}$ is an arbitrary constant time. Judging from the reduced variables at the limiting cases of small rate of shear and steady flow, one may have to keep $t / a_{\mathrm{T}}$ or $\kappa a_{\mathrm{T}}$ constant in 
Flow Properties of Polymer Solutions. I.

order to establish a shear rate-temperature or a time-temperature reduction rule, respectively. We studied this possibility by keeping $t / \eta^{0}$ constant and examining the shear rate-temperature reduction rule. This procedure is equivalent to that of the study of the shear rate-temperature reduction rule, because $T_{0} \rho_{0} / T_{\rho}$ is not very sensitive to variation of $T$ and $\eta_{0}{ }^{0}$ is a constant with respect to varying $T$.

Figure 6 shows $\tilde{\eta}(t, \kappa) / \eta^{0}$ obtained at various temperatures for $t / \eta^{0}=0,10^{-5}$, and $10^{-4} \mathrm{sec} /$ poise plotted against $\kappa a_{\mathrm{T}}$, where the reference temperature is $30^{\circ} \mathrm{C}$ and $a_{\mathrm{T}}=\eta^{0} / \eta_{0}{ }^{\circ}$. The result for $t / \eta^{0}=0$ is that for the steady-shear viscosity as discussed above. It may be obvious that $\tilde{\eta}(t, \kappa) /$ $\eta^{0}$ at $t / \eta^{0}=10^{-5}$ or $10^{-4}$ is also reduced to a single function when $\kappa a_{\mathrm{T}}$ is chosen as variable. Thus the shear rate-temperature reduction rule is applied not only to $\eta(\kappa)$ but also to $\tilde{\eta}(t, \kappa)$ at constant $t / \eta^{0}$. It may be noted that $\tilde{\eta}(t, \kappa) / \eta^{0}$ decreases more rapidly with increasing $\kappa$ when $t / \eta^{0}$ is larger.

Figure 7 shows the plot of $\bar{\eta}(t, \kappa) / \eta^{0}$ for $t / \eta^{0}=$ $3 \times 10^{-5}$ and $9 \times 10^{-5} \mathrm{sec} /$ poise obtained at various temperatures against $\kappa a_{\mathrm{T}}$, where the reference temperature is $30^{\circ} \mathrm{C}$ and $a_{\mathrm{T}}=\eta^{0} / \eta_{0}{ }^{0}$. The result for the steady-shear viscosity, or $\bar{\eta}(t, \kappa)$ at $t / \eta^{0}=$ $\infty$, is also shown with a thick line. Obviously $\bar{\eta}(t, \kappa) / \eta^{0}$ obtained at various temperatures is a

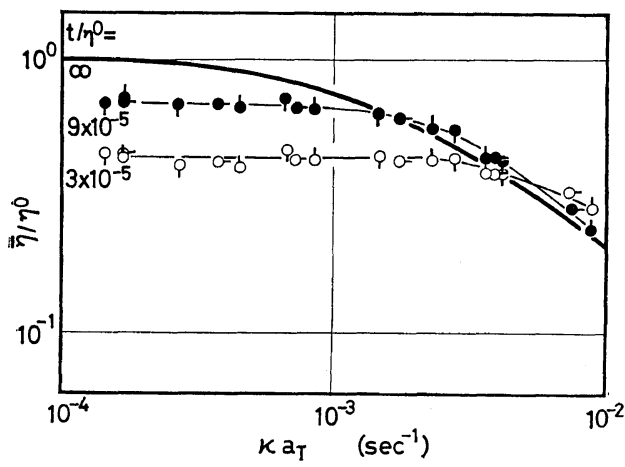

Figure 7. Reduced stress development function $\bar{\eta}(t, \kappa) / \eta^{0}$ plotted against reduced rate of shear $\kappa a_{\mathrm{T}}$ for solution of polystyrene in chlorinated biphenyl. Reference temperature is $30^{\circ} \mathrm{C}$ and various directions of "pips" represent temperatures as indicated in Figure 5. Reduced times $t / \eta^{0}$ are infinity for thick line, $9 \times 10^{-5} \mathrm{sec} /$ poise for filled circles, and $3 \times 10^{-5} \mathrm{sec} /$ poise for open circles. unique function of $\kappa a_{\mathrm{T}}$ if compared at any constant value of $t / \eta^{0}$. The quantity $\bar{\eta}(t, \kappa) / \eta^{0}$ at $t / \eta^{0}=10^{-5} \mathrm{sec} /$ poise (not shown in Figure 7) is independent of $\kappa a_{\mathrm{T}}$ in the range of rate of shear investigated in this study. The curves for various values of $t / \eta^{0}$ intersect one another in the range of high reduced rate of shear reflecting the phenomenon of overshoot of $\bar{\eta}(t, \kappa)$ in Figure 2.

\section{Conclusion}

It has been revealed that the shear ratetemperature reduction rule is applicable to $\bar{\eta}(t, \kappa)$ and $\tilde{\eta}(t, \kappa)$ obtained at various temperatures if compared at a constant value of reduced time $t / a_{\mathrm{T}}$. This result implies in return that the time-temperature reduction rule is applicable with the use of the same shift factor $a_{\mathrm{T}}$ if compared at a constant value of reduced rate of shear $\kappa a_{\mathrm{T}}$. In other words, $\bar{\eta}(t, \kappa) / \eta^{0}$ or $\tilde{\eta}(t, \kappa) / \eta^{0}$ is a single-valued function of two variables $t / a_{\mathrm{T}}$ and $\kappa a_{\mathrm{T}}$. Thus the method of reduced variables may be employed to obtain data of $\bar{\eta}(t, \kappa)$ or $\tilde{\eta}(t, \kappa)$ over wide ranges of $\kappa$ or $t$ for polystyrene solutions in chlorinated biphenyl. It may be noted that an identical shift factor $a_{\mathrm{T}}$ determines the effect of varying temperature on time $t$ and rate of shear $\kappa$. This result seems to indicate that the molecular process involved in the nonlinear behavior as well as in the relaxation behavior of linear viscoelasticity is controlled by the motion of polymer segments, since $a_{\mathrm{T}}$ is supposed to represent the temperature dependence of the segmental friction coefficient. ${ }^{15}$ The reduction rule revealed above may put some restriction on possible choice of parameters in constitutive equations. In giving a definite functional form to a rate-dependent relaxation spectrum, ${ }^{6}$ for example, one has to employ an identical basic time constant in the coefficients of the relaxation times and the rate of shear.

Acknowledgment. We are indebted to Iwamoto Seisakusho Co., Ltd., for great help in constructing the apparatus.

\section{REFERENCES}

1. G. V. Vinogradov and I. M. Belkin, J. Polym. Sci. Part A, 3, 917 (1965). 
2. A. Ya Malkin, B. V. Yarlykov, and G. V. Vinogradov, Rheol. Acta, 9, 329 (1970).

3. J. D. Huppler, I. F. Macdonald, E. Ashare, T. W. Spriggs, and R. B. Bird, Trans. Soc. Rheol., 11, 181 (1967).

4. I-J. Chen and D. C. Bogue, ibid., 16, 59 (1972).

5. M. Sasaki, H. Fukaya, and M. Nagasawa, ibid., 16, 635 (1972).

6. See for example, M. Yamamoto, "Buttai no Henkeigaku," Seibundo-Shinkosha, Tokyo, 1972.

7. B. Bernstein, E. A. Kearsley, and L. J. Zapas, Trans. Soc. Rheol., 7, 391 (1963).

8. L. J. Zapas and T. Craft, J. Res. Nat. Bur. Stand. A, 69, 541 (1965).

9. Y. Einaga, K. Osaki, M. Kurata, S. Kimura, and M. Tamura, Polymer J., 2, 550 (1971).

10. Y. Einaga, K. Osaki, M. Kurata, S. Kimura, N. Yamada, and M. Tamura, ibid., 5, 91 (1973).
11. M. Yamamoto, Trans. Soc. Rheol., 15, 331, 783 (1971).

12. K. Osaki, Y. Einaga, M. Kurata, N. Yamada, and M. Tamura, Polymer $J$. , in press.

13. M. Yamamoto, Zairyo, 21, 355 (1972).

14. See for example, J. D. Ferry, "Viscoelastic Properties of Polymers," 2nd ed., John Wiley \& Sons, Inc., New York, N.Y., 1970, Chapter 3.

15. See Chapter 11 of ref 14 for references.

16. See Chapter 14 of ref 14 for references.

17. Y. Einaga, M. Kurata, and M. Tamura, to be published.

18. S. Ohta, Y. Einaga, K. Osaki, and M. Kurata, Bull. Inst. Chem. Res. Kyoto Univ., 51, 220 (1973).

19. Y. Einaga, K. Osaki, M. Kurata, and M. Tamura, Macromolecules, 4, 87 (1971); 5, 635 (1972).

20. Y. Einaga, K. Osaki, M. Kurata, and M. Tamura, ibid., 6, 598 (1973). 